AperTO - Archivio Istituzionale Open Access dell'Università di Torino

\title{
Mathematical modelling with technology: the role of dynamic representations
}

\section{This is the author's manuscript}

Original Citation:

Availability:

This version is available http://hdl.handle.net/2318/92280

since 2019-09-08T11:06:05Z

Published version:

DOI:10.1093/teamat/hrr027

Terms of use:

Open Access

Anyone can freely access the full text of works made available as "Open Access". Works made available under a Creative Commons license can be used according to the terms and conditions of said license. Use of all other works requires consent of the right holder (author or publisher) if not exempted from copyright protection by the applicable law. 


\section{(5) \\ UNIVERSITÀ DEGLI STUDI DI TORINO}

This is an author version of the contribution:

Questa è la versione dell'autore dell'opera:

Arzarello, F., Ferrara, F. \& Robutti, O. (2012). Teaching Mathematics and Its Applications, 31(1), 20-30. DOI: 10.1093/teamat/hrr027

The definitive version is available at:

La versione definitiva è disponibile alla URL:

http://teamat.oxfordjournals.org/content/31/1/20.full.pdf+html 


\title{
Mathematical modelling with technology: the role of dynamic representations
}

\author{
Ferdinando Arzarello, Francesca Ferrara and Ornella Robutti* \\ Dipartimento di Matematica, Università di Torino
}

*Email: ornella.robutti@unito.it

\begin{abstract}
In this research we present the use of some technologies in problem solving activities (at different secondary school grades), aimed at finding a model for a geometric configuration, and representing this model in various ways: through a construction, through a Cartesian graph, etc. The task is part of a teaching experiment, in which students used paper and pencil, and technological tools: a sensor and a calculator (at a lower grade), GeoGebra and TI-Nspire (at a higher grade). We show results in terms of the passage from static to dynamic representations and back, to observe how technology may foster dynamic thinking for students solving mathematical problems. Data suggest that the dynamic features of technology support the genesis of conjectures, and their validation or refutation, along with the choice of independent and dependent variables. Results are used to prepare materials for teacher training in an e-learning Moodle platform (Comenius EdUmatics Project).
\end{abstract}

\section{Introduction}

Representations in mathematics education have been studied since a long time, but particularly in the last decades, when technology offers many kinds of representations (graphic, symbolic, numeric and so on). The representations available in the technological tools are different from some years ago, when a prevalence of static characters constituted the scene, as final products of specific computer processing (e.g. a table in a spreadsheet, a graph in a programming language like Pascal; see Ferrara et al., 2006).

Nowadays, more advanced forms of representation have been created. Dynamicity is an intrinsic feature of tools, and is also present on the screen of a computer or a calculator. Examples are given by: a geometric figure constructed in Dynamic Geometry Software (DGS); a graph of a surface traced through a symbolic software like Mathematica; a function represented using an i-Pad application. All of these mathematical objects can be dragged, enlarged, turned, viewed from different perspectives. The new representational infrastructures have consequences on learning too (Kaput et al., 2002; Hegedus \& Moreno-Armella, 2009; Sträßer, 2009), giving a fundamental support for exploring, discovering, conjecturing in mathematical problems where processes changing over time (dynamic representations) must be considered. In such cases, dynamic representations allow cognitive affordances that may lighten solvers' cognitive load according to the specific type of representation used (see for example Ainsworth \& Van Labeke, 2004). 
Since conjecturing using dynamic thinking is also possible without technology (Simon, 1996), this does not mean that poorer materials (like paper and pencil) just permit for static representations that of course may also have dynamic features. Sketches by learners are a case: they are powerful given their iconic character, and acquire dynamic nature in the moment students interact with and use them to communicate thoughts. Simply, the modern infrastructures give students new possibilities that previous generations students did not have, for developing a type of transformational (dynamic) reasoning at primary, secondary and university level.

Furthermore, the use of dynamic representations in modelling situations enhances the dialectic between the empirical side and the theoretical side of the mathematical objects. For example, Data Capture function in TI-Nspire (used to model a situation) allows data collections in a way similar to physical samples (Arzarello, 2009). On the other hand, DGS enable students to choose various quantities as independent variable, supplying different dynamic representations of one situation, and the exploration of invariants in the corresponding mathematical objects. As R. Noss said in his plenary lecture at ICTMT10, technology may help students in their activity, 're-representing ideas of change with a medium that supports change'.

\section{The project}

EdUmatics is a Comenius Project aimed at preparing materials of lifelong learning for in-service teachers across Europe (EdUmatics, 2009), and using them in presence (by means of congresses, seminars, workshops,...) and at a distance (through a Moodle platform for e-learning: forum, activities, wiki,...). Our part in the project is to construct, with a French partner (University of Lyon), a section devoted to static and dynamic representations (Aldon, 2010), used in a fruitful way to solve problems and model situations. In this section, we prepared a problem on sequences of natural numbers and a problem on geometric modelling, while the French partner prepared a problem on statistics. The activities were carried out in Italian and French schools to experience the role of technology in real classroom contexts. We focus here on the geometric activity. 'The walker problem' proposes some situations about the motion of a constant speed walker moving along the perimeter of a geometric figure. It asks students to set up a model describing the variation of the dependent variable (the walker distance from the centre of the figure) as a function of an independent variable, given or not, according to the school grade. Learners are first invited to imagine, recognize and describe quantities in the geometric situation individually, then in small groups, just with paper and pencil, before making calculations or using technology. Starting from the observation in the particular case of the circle (Fig. 1), where the dependent variable (the radius) is invariant to changes of the independent variable (the angle or the arc), students come to consider the more complex case of a square (Fig. 2). When working in groups, students have to fill in a paper-sheet, and then they participate to a classroom discussion coordinated by the teacher.

The walker problem in the case of the circle (Fig. 1)

$\mathrm{Mr}$ Bean moves at a constant speed along a circle with centre $\mathrm{O}$ and given radius $\mathrm{r}$, starting from a point $\mathrm{A}$ on the circle.

Mr Bean wants to describe how his distance from the centre $\mathrm{O}$ of the circle changes, during his moving along the circle. How can you help him?

The walker problem in the case of the square (Fig. 2)

$\mathrm{Mr}$ Bean moves at a constant speed along a square $\mathrm{ABCD}$ with centre $\mathrm{O}$ and given side, starting from a point $\mathrm{P}$ on the square. 


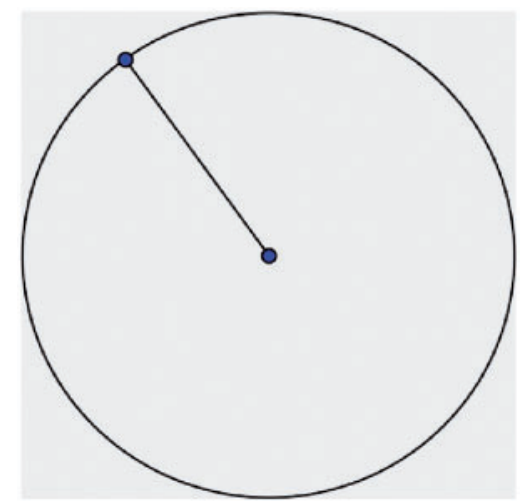

FIG. 1. Case of the circle. This figure appears in colour in the online version of Teaching Mathematics and its Applications.

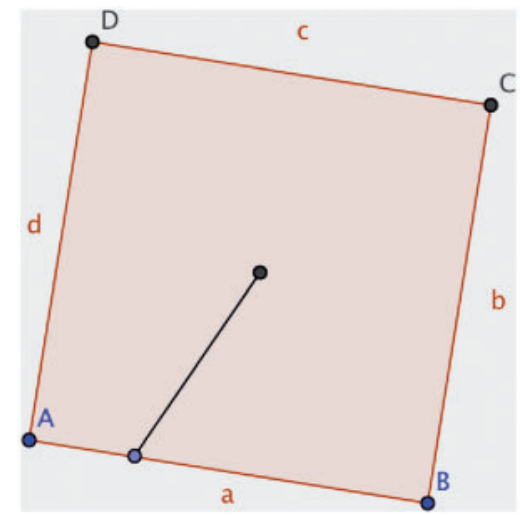

Fig. 2. Case of the square. This figure appears in colour in the online version of Teaching Mathematics and its Applications.

$\mathrm{Mr}$ Bean wants to describe how his distance from the centre $\mathrm{O}$ of the square changes, during his moving along the square. How can you help him?

'The walker problem' has been implemented at different secondary grades, and technological tools of various kinds were also used. At lower secondary school (grade 8), students first utilize a motion sensor and a graphic calculator to observe the graph of the situation, then they construct and represent the model in GeoGebra. At higher secondary school, grade 10 students use GeoGebra with a free choice of the independent variable; grade 11 students use TI-Nspire to find the model (although the choice of the independent variable is suggested by the teacher). Different tools give different possibilities to represent and to work with the model of the situation, but they also have some common features. We show here some excerpts from the videotaping of the classroom activities carried out during plain school time. The methodology was that of mathematics laboratory (Arzarello \& Robutti, 2010). The filmed students attend different classes and schools, their teachers participated in the project as experimenters, and a master degree student was the observer. 


\section{Dynamic representations}

The first excerpt is taken from the 8th grade classroom activity. Students are thinking of the problem in the case of the square, and discussing in their group (before using the motion sensor), while the observer films them sometimes interacting with them.

Observer: You told me that distances change. Why?

Paolo: From the centre to A (distance) will be 1,5, but from $\mathrm{P}$ to $\mathrm{C}$ since it is not a line anymore... say, it is always a straight line, but the angle is not $90^{\circ}$ anymore, and it changes, now here it is not proper at the half (between A and B).

Gabriele: Hmm, no, 'cause here you are not anymore... here he is going away (his finger follows the segment $\mathrm{AB}$ and goes over, passing $\mathrm{B}$ ).

Paolo: He's going far and far.

Observer: Ok, here he's going away, far and far. Then what distance does he measure?

Paolo: It is always ... greater.

Gabriele: Greater till arriving at B (with his pen he follows the segment AB and he stops in B).

Paolo: Here (at point B) will be the greatest point.

Observer: And then?

Paolo: In the four angles...

Gabriele: And then it will decrease till arriving at the middle point of the side (with his pen he follows the side subsequent to $\mathrm{AB}$ ), and he will find the same distance as PA.

The students come to understand that the function is not constant as in the case of the circle, but it changes its values from a maximum, at each vertex of the square, to a minimum, at the middle point of every side. At this moment, they are not aware of the shape of the function graph, and they sketch it with a piecewise graph: four straight lines going up and down connected with each other. Only after the experiment with sensor and calculator (Fig. 3), they are able to understand the actual shape of the graph, made of the same curved piece repeated four times (Fig. 4).

The second excerpt comes from 10th grade students working with GeoGebra. They conjecture on and construct the figure relative to the circle, then they approach the square problem: some groups choose the angle as independent variable, other groups the path covered along the square. In both cases, the model is a periodic function (with period given by $90^{\circ}$ or one side), whose graph is a decreasing and increasing curve that can resemble a parabola (they find it through the locus function of the software). In order to investigate what kind of curve it is, the students select five points on the first piece of the curve (with the angle as variable). Asking the DGS to find a conic passing through these points, it gives an ellipse (Fig. 5). However, trying to calculate the solution symbolically, the students discover that the model is the inverse of a cosine, instead of an ellipse.

This mismatch offers the teacher a chance for discussing on the approximation by the software to look for a conic interpolating the five points, and on the necessity to always check the solution the software provides, comparing it with that theoretically found in paper and pencil. By the way, the approximation is easily revealed by zooming on the graph, and by the fact that moving the points the symbolic expression of the ellipse changes.

Teacher: Moving the points (on the locus), instead of those points, you take other five points, on the same arc, the ellipse modifies ... This already tells us something. What does it happen? What does it mean that given five points I get an ellipse, moving the five points in another place, again on that locus, I get a new ellipse, with another equation? It means I'm having approximations, it 


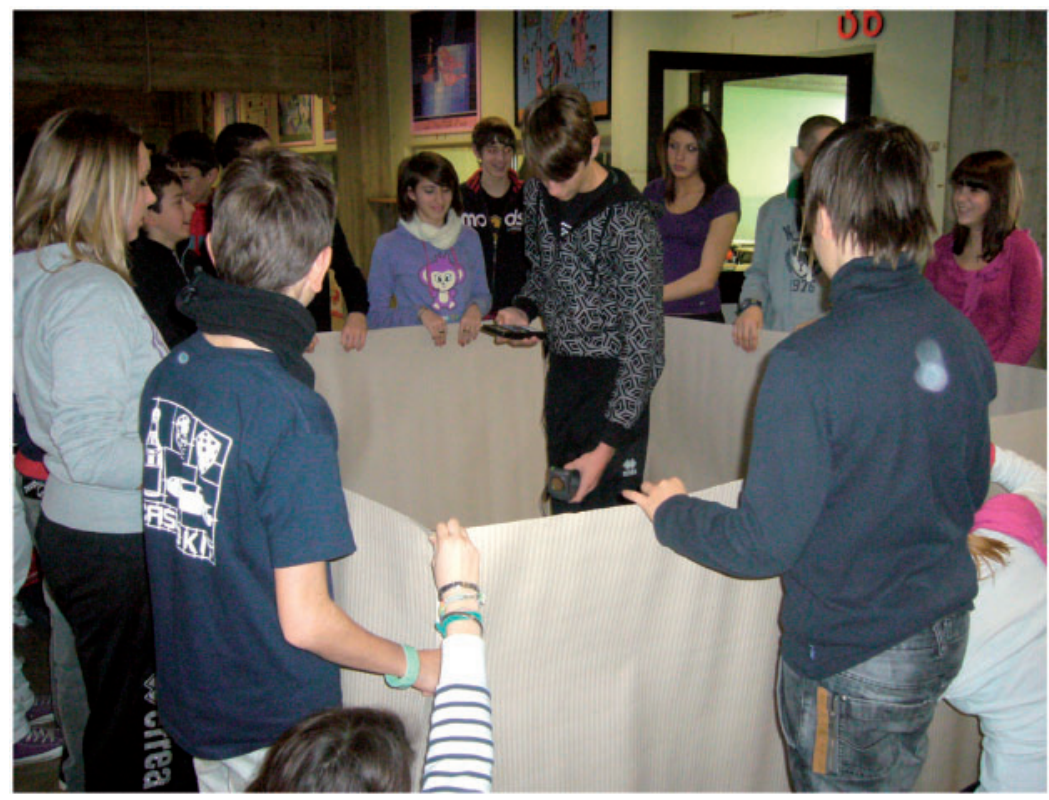

FIG. 3. Use of the motion sensor. This figure appears in colour in the online version of Teaching Mathematics and its Applications.

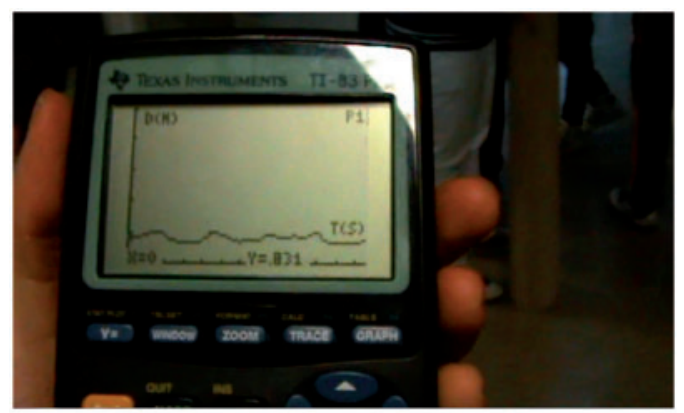

Fig. 4. Graph by the sensor. This figure appears in colour in the online version of Teaching Mathematics and its Applications.

means this arc here is not exactly the first ellipse, nor the second, nor the third one ... maybe, it's not even an ellipse, but the approximations of GeoGebra, to find a curve through those five points we chose, makes it to become an ellipse.

Choosing the path as independent variable, students obtain another periodic function, and again they select five points on the curve to get a model. GeoGebra gives them a piece of a hyperbola (Fig. 6), which can be calculated applying Pythagoras' theorem. In this case, the symbolic manipulation furnishes a model matching that obtained with the software. 


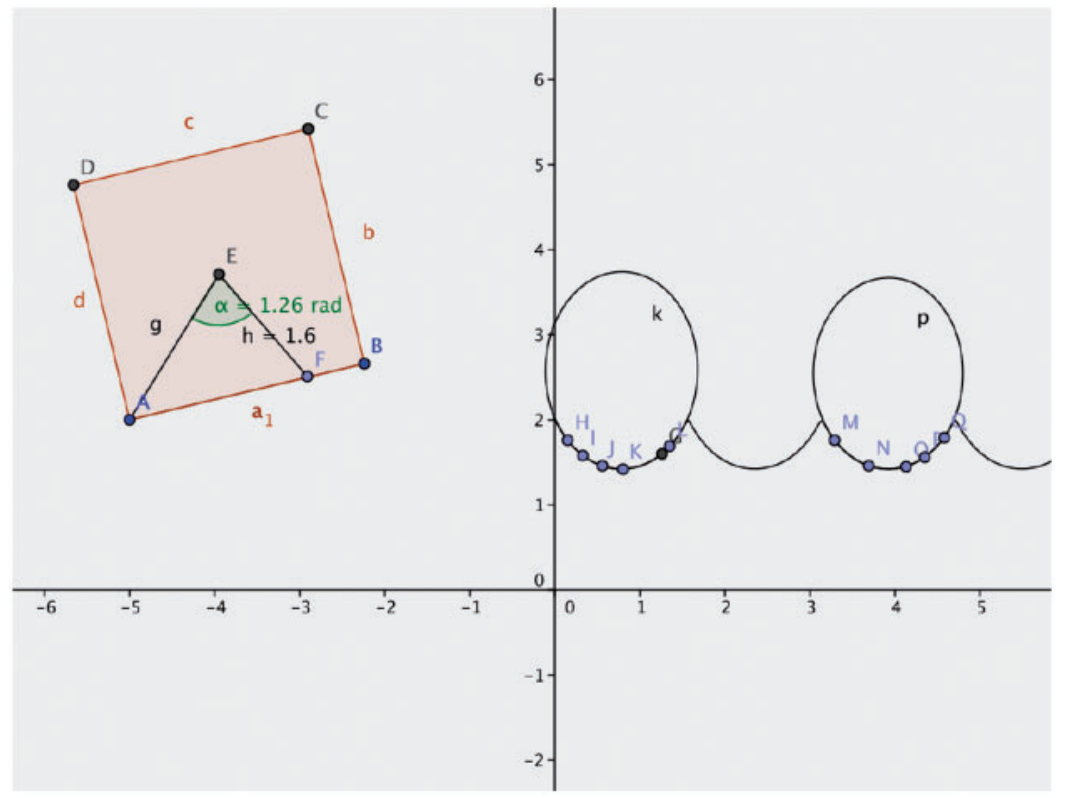

FIG. 5. Angle as variable. This figure appears in colour in the online version of Teaching Mathematics and its Applications.

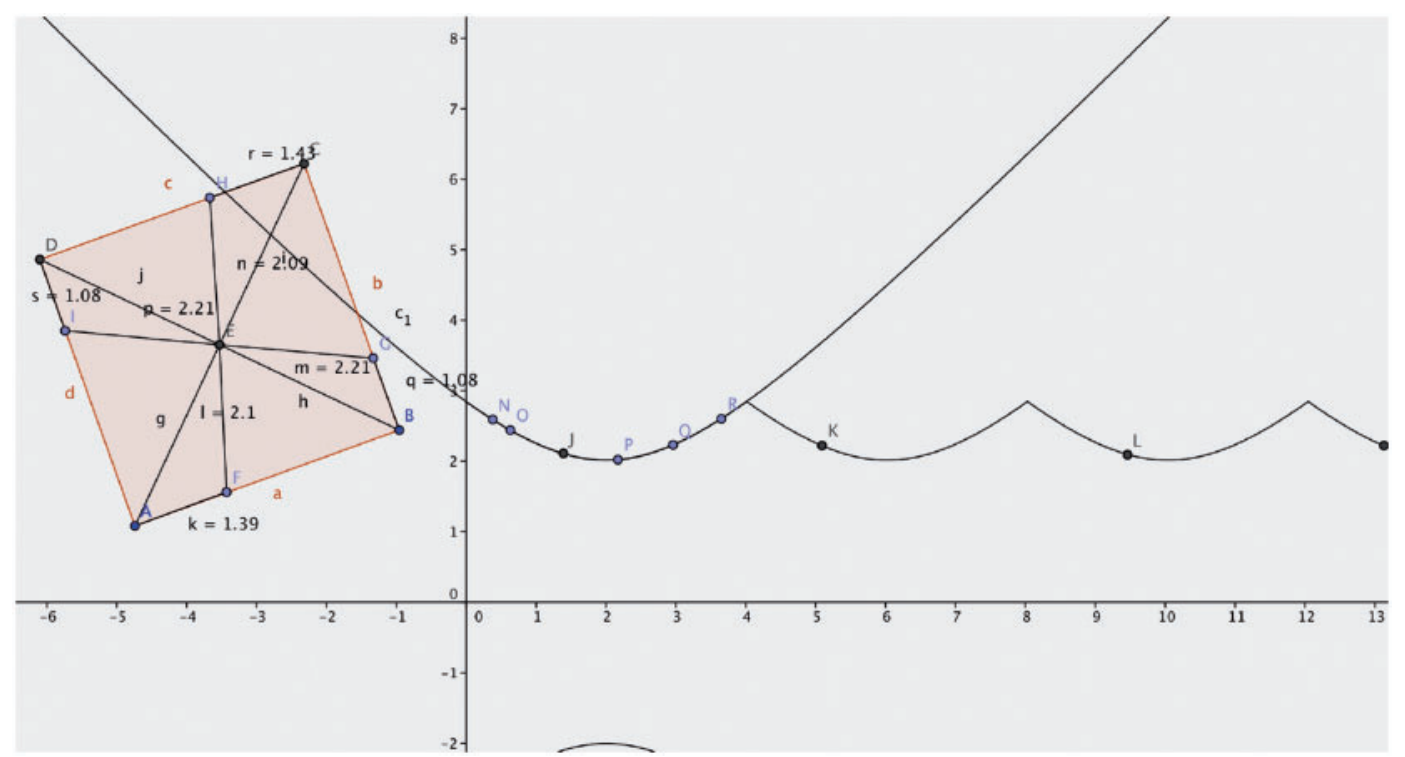

FIG. 6. Path as variable. This figure appears in colour in the online version of Teaching Mathematics and its Applications. 
The third excerpt refers to 11th grade students, who solve the same problem as 10th grade students, using TI-Nspire and TI-Navigator. In the following, a part of group activity in paper and pencil, before using technology, is presented.

Emanuela: Then, practically it is ... then ...

Alessandro: A zig-zag (miming it in the air)... let's fix the side equal to 1.

Alex: It starts from the maximum...

Alessandro: Yeah ... $\frac{\sqrt{2}}{2} \ldots$ cause it is at the middle of the diagonal ... and it arrives at $\frac{1}{2}$.

The students' reasoning on the activity in paper and pencil is, corroborated by imagination, more complex than the one of the 8th grade students, and makes also use of measures and quantitative relations among the square's sides. Nevertheless, at their first approach they come to sketch a zig-zag function as well as 8th grade students did (Fig. 7).

Teacher: What do you want to verify?

Emanuela: We would like to verify if PO (namely the distance from the centre) constantly decreases.

Teacher: When you say constantly ... that is, are you thinking linearly?

Emanuela: Yeah.

Teacher: So, pass to use TI-Nspire.

Students are used to analyse a function in terms of variation of its values, and ways of variation, because they study the mathematics of change since grade 9, using different representations of functions and different technologies. The intuition on this problem guides them to infer that the model of the situation is made of linear functions connected together. Using then the TI-Nspire handheld technology, students want to check if their conjecture is true or not. With TI-Nspire, they construct a square and connect a point on the square with its centre through a segment, calculating this distance. In

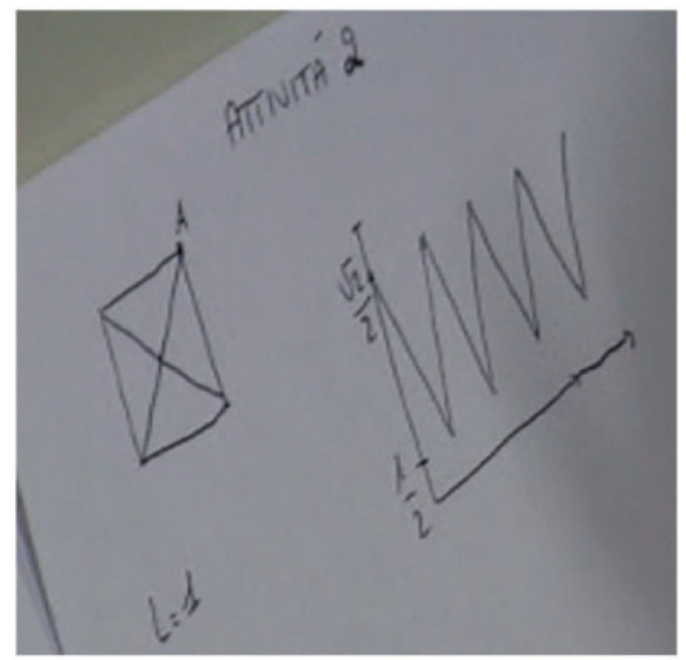

Fig. 7. Graph in paper and pencil. This figure appears in colour in the online version of Teaching Mathematics and its Applications. 


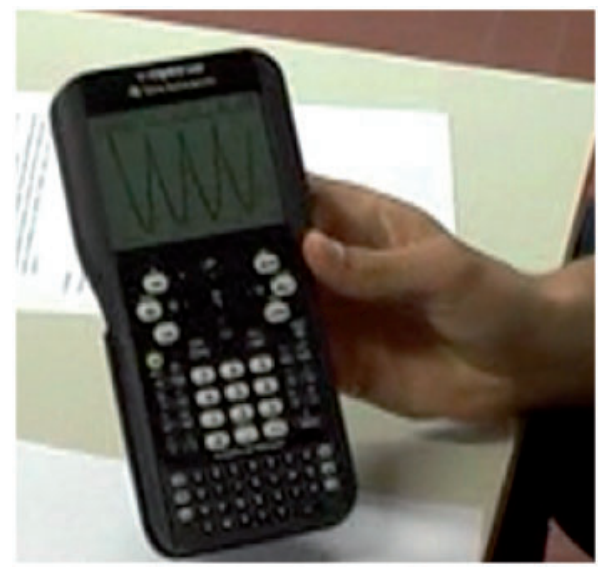

FIG. 8. Graph on the calculator. This figure appears in colour in the online version of Teaching Mathematics and its Applications.

the spreadsheet environment, they collect data for this distance capturing it as variable from the geometric figure, and automatically receive data from the figure in function of a counter that has the invisible meaning of time (a natural sequence from 1 to 400). Simultaneously, in the graphic environment, they have the possibility to see the model animating the independent variable (Fig. 8).

Teacher: Did it confirm your conjecture or not?

Alessandro: Yeah.

Emanuela: No, we thought that...

Alex: It confirms the doubt.

Teacher: What was the doubt? How could you see if it was linear or not?

Alex: Differences...

Teacher: Differences, yeah? With first and second differences. First differences, ok ... if they are constant it is linear, isn't it? You could do the first and second differences.... are they or not pieces of parabola, in your opinion?

Alex: According to us ... yeah.

Teacher: Yeah, then how could you verify if they are pieces of parabolas?

Alessandro: With the second differences.

The students calculate first and second differences and observe that the last ones are not constant. So they come to the conclusion that it cannot be a parabola, and they decide to go on finding differences, in order to discover if, at a certain point, they are constant. The teacher, through the discussion, outlines that it could happen that they do not find constant differences, as in the case of a non-polynomial function: hence, they are finally able to acknowledge that this is the case.

\section{Discussion}

To approach the task, all the students move from the personal senses (Leontiev, 1994) they attach to the situation to making conjectures on a possible mathematical model. Regardless of the different kinds of technology used, most students (at all levels) first conjecture that in the case 
of the square the model is linear. This is not in contrast with the usual manner we all, included secondary school students, think in everyday life, since, at a first approximation, the world is interpreted as linear (De Bock et al., 2007). Then technology is used to test the conjectures: these may be more or less effective for properly modelling the situation. Hence, different interventions of the teacher are necessary to support the evolution of students' personal senses towards a shared scientific one. Such behaviour is generalized throughout the grades. The feedback of the tools and the modulation of the teacher's interventions also depend on the classrooms' mathematical knowledge and culture. All these components (technology, teacher, classroom culture) play a fundamental role in supporting the students to elaborate new conjectures about the mathematical nature of the graph.

The case of the finite differences for the 11th grade students working with TI-Nspire is emblematic. They are used to calculate finite differences, in the classroom practice, as a means for understanding the degree of a polynomial function. This practice constitutes a background competence for their mathematical work on functions. During the activity on 'The walker problem', they recover and apply it to the particular case of the square, in order to validate or confute their conjectures. In this manner, the students go back and forth between static and dynamic representations (the zig-zag shape mimed by Alessandro's gestures in the air becomes the piecewise graph traced on paper; then, such a graph is compared with that obtained on the calculator through the animation, according to which the finite differences are founded). However, this kind of behaviour points out a limit of students' reasoning: indeed, they implicitly assume that the finite difference strategy will work in any case (only knowing that it does for polynomials). There is a contrast here between the personal sense and the scientific one, according to which the right model is not a polynomial. To overcome the contrast, the teacher's intervention is essential: he is able to prompt the students that not all functions in the end have a constant $n$-th difference table and thus to accept within their conjecturing horizon the possibility of a non-polynomial function as a model of the problem. In a Vygotskijan perspective, he triggers the evolution of students' personal senses towards a shared scientific sense: the mathematical discussion he orchestrates in the classroom is the means by which he is able to manage such mediation.

Another aspect of the teacher's eventual interventions is shown by the grade 10 students using GeoGebra. Once the groups have got the graph that models the walker problem, they conjecture that it is made of arcs of hyperbola. Now they use the software to validate this finding. They know that given five points, the software is able to draw the conic passing through them. Hence, the students choose five points on the graph: but in one case (when the independent variable is the angle) they get an ellipse, against their expectation. Of course this is just an approximation of the actual model. Again, in this situation the intervention of the teacher is relevant to help the students to find the algebraic expression of the functions describing the geometrical situation (respectively, a hyperbola and $\cos ^{-1}$, depending on the independent variable chosen) and to share the work of the filmed small group with the whole classroom. The classroom discussion opens then a cognitive space where all the strategies and the solutions are shared, and students can discuss the comparison between the choices of one independent variable in place of another one in terms of the model. Anew, the teacher makes apparent a bridge between students' personal senses and the scientific sense. It is not the case that the personal senses of the students are cancelled; on the contrary, they may evolve in new senses closer and closer to the mathematical ones.

In relation to the choice of the independent variable, an interesting point may be outlined with respect to producing a deeper cognitive bridge. In fact, we involved some University students attending a course in Mathematics Education (for prospective mathematics teachers) in 'The walker problem' (without filming them). We could orchestrate a very rich discussion on the different types of the 
phenomenon described by one choice or another of the independent variable. Taking the angle as independent variable gives rise to the model relative to a constant angular velocity motion, while the path as independent variable corresponds to the model for a constant linear velocity motion. Namely, the two models concern different physical motions. This is relevant regarding the expected shape of the graph, and as such may affect students' conjectures and their checking.

In the course of the project (EdUmatics, 2009), another activity was given to the students, in the arithmetic context of sequences of natural numbers. The aim of the activity was again to find a model for different sequences starting from some of their numbers, and to represent the model in various ways: using a table, a recursive function, a close formula. Despite the difference of the tool used (in this case, a spreadsheet), we noticed that a continuous passage from static to dynamic representations and back is still present. Moreover, the spreadsheet fosters students to think dynamically, adopting dynamic strategies in the search for the formula.

\section{REFERENCES}

Ainsworth, S. \& Van Labeke, N. (2004) Multiple forms of dynamic representation. Learn. Instr., 14, 241-255.

Aldon, G. (2010) Handheld calculators between instrument and document. [The Role of Handheld Technology in the Mathematics Classroom (P. Drijvers \& H. G. Weigand eds)]. ZDM Math. Educ., 42, 733-745.

Arzarello, F. (2009) New technologies in the classroom: towards a semiotics analysis. Relatively and Philosophically Earnest: Festschrift in Honor of Paul Ernest's 65th Birthday (B. Sriraman \& S. Goodchild eds). Chishing, Charlotte, NC: Information Age Publishing, Inc., pp. 235-255.

ArZarello, F. \& Robutti, O. (2010) Multimodality in multi-representational environments. ZDM Math. Educ., 42, 715-731.

De Bock, D., Van Dooren, W., Janssens, D. \& Verschaffel, L. (2007) The Illusion Of Linearity: From Analysis to Improvement, Mathematics Education Library, vol. 41. New York: Springer.

EdUmatics (2009) European Development for the Use of Mathematics Technology in Classrooms, 503254-LLP-1-2009-1-UK-COMENIUS-CMP. Application form.

Ferrara, F., Pratt, D. \& Robutti, O. (2006) The role and the use of technologies for the teaching of algebra and calculus. Handbook of research on the psychology of mathematics education (A. Gutierrez \& P. Boero eds). Rotterdam: Sense, pp. 237-273.

Hegedus, S.J. \& Moreno-Armella, L. (2009) Intersecting representation and communication infrastructures. ZDM Math. Educ., 41, 399-412.

Kaput, J., Noss, R. \& Hoyles, C. (2002) Developing new notations for a learnable mathematics in the computational era. Handbook of International Research in Mathematics Education (L. D. English, M. Bartolini Bussi, G. A. Jones, R. A. Lesh \& B. Sriraman eds), 2nd edn. New York and London: Routledge, pp. 693-715.

Leontiev, D.A. (1994) The concept of personal sense through the ages. Multidisciplinary Newsletter for Activity Theory, 15/16, 9-12.

Simon, M. (1996) Beyond inductive and deductive reasoning: the search for a sense of knowing. Educ. Studies Math., 30, 197-210.

STRÄßER, R. (2009) Instrument for learning and teaching mathematics: an attempt to theorise about the role of textbooks, computers and other artefacts to teach and learn mathematics. Proceedings of the 33rd Conference of the International Group for the Psychology of Mathematics Education (M. Tzekaki, M. Kaldrimidou \& H. Sakonidis eds), vol. 1. Thessaloniki, Greece: Aristotle University of Thessaloniki \& University of Macedonia, pp. 67-81. 
Ferdinando Arzarello is Full Professor at the Department of Mathematics of the University of Torino, in Italy. He is President of the European Society for Research in Mathematics Education (ERME). He has been Head of the Department of Mathematics, of the Doctoral School in Mathematics, of the Teacher Training School in Piedmont. He is involved in teacher education in national projects in Italy (m@t.abel, PON).He has been invited speaker in many national and international conferences. He has been and is currently engaged in many national and international Projects and collaborations. He has published many articles in national and international journals and books.

Francesca Ferrara is Assistant Professor at the Department of Mathematics of the University of Torino, in Italy. She took her Master degree in Mathematics in 2001, and her Ph.D in Mathematics in 2006. Her area of expertise is Mathematics Education, on which both the dissertations were discussed. She is part of the scientific committee of the National Seminar on Research in Mathematics Education that takes place each year in Italy. She has been and is currently engaged in many national and international Projects and collaborations. She has published in national and international journals and books.

Ornella Robutti is Associate Professor in Mathematics Education at the Department of Mathematics of the University of Torino, in Italy. She took her Master degree in Mathematics in 1984 and her Master degree in Physics in 1989 at the University of Torino. She is involved in teacher education in national projects in Italy (m@t.abel, PON). She is the person in charge of the GeoGebra Institute of Torino and of the project DIFIMA in Rete. She is the author of many publications in the field of Mathematics Education as well as high school books of Physics and Mathematics. Her field of research is the teaching and learning processes in Mathematics with the support of technologies. 